\title{
Analytical modeling of mechanical behavior of structures: Comparative analysis, experimental validation and numerical correction with FEM of material defects generated by anisotropic etching
}

\author{
Hicham Bourouina $^{1, \mathrm{a}}$, Réda Yahiaoui ${ }^{2}$, Elmar Yusifli ${ }^{3}$, Mohammed El Amine Benamar ${ }^{1}$ \\ and Abdellah Sahar ${ }^{1}$ \\ ${ }^{1}$ Physics department, University of Blida, 09000 Blida, Algeria \\ ${ }^{2}$ Femto-st Institute, University of Franche Comté, 25000 Besancon, France \\ ${ }^{3}$ NanomedecineLab, University of Franche Comté, 25000 Besancon, France
}

\begin{abstract}
This paper presented semi-analytical approach in order to investigate the influence of manufacturing process defects on the elasticity of thin microbeam. The Rayleigh Beam Model (RBM) will be analyzed and corrected using 3D FEM scripts including the effects of the cross-section shape and the under-etching. This model was tested on measurements of thin chromium microbeam of dimensions $\left(80 \times 2 \times 0.95 \mu \mathrm{m}^{3}\right)$. The results show that the influence of defects is very significant for the extracted value of Young's modulus where it is very close to the measured value and it is about $279.1 \mathrm{GPa}$.
\end{abstract}

\section{Introduction}

Measurement of the resonant frequencies is a common technique for the evaluation of the Young's modulus of thin microbeams. However, the measured frequencies are very sensitive to the under-etching and the cross-section shape due to the manufacturing process [1]. The improved model proposed in this study investigates these defects for an accurate determination of the Young's modulus value.

\section{Improved modelling including manufacturing process defects}

The single clamped microbeam may be described by the Rayleigh Beam model (RBM) which takes into account the rotation effect of cross-section [2]. The finite elements method (FEM) is performed to determine the corrective factors which must be applied to investigate the influence of the manufacturing process defects on the resonant frequency. This later can better be given by:

$$
f_{n}^{r}=\frac{1}{2 \pi} \sqrt{\left(a_{n}^{r^{2}}-b_{n}^{r^{2}}\right)\left\{\left[E\left(1+\alpha\left(v^{2} /\left(1-v^{2}\right)\right)(1+\beta)\right)\right] /\left(\rho L^{2}(1+\gamma)^{2}\right)\right\}} .
$$

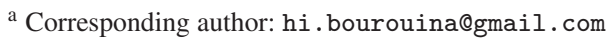

This is an Open Access article distributed under the terms of the Creative Commons Attribution License 4.0, which permits unrestricted use, distribution, and reproduction in any medium, provided the original work is properly cited.
} 


\section{MATEC Web of Conferences}

Table 1. The first three values of Young's modulus calculated from the measured frequencies.

\begin{tabular}{|c|c|c|c|}
\hline Vibration & $f[\mathrm{KHz}]$ & \multicolumn{2}{|c|}{ Classical model } \\
\cline { 3 - 4 } modes & measured & EBM [1] & RBM \\
\hline $1^{\text {st }}$ mode & 0135.000 & 227.9022 & 264.6028 \\
\hline $2^{\text {nd }}$ mode & 0839.500 & 224.3974 & 277.3457 \\
\hline $3^{\text {rd }}$ mode & 2317.000 & 218.0236 & 245.7785 \\
\hline
\end{tabular}

Table 2. The values of Young's modulus calculated from the first three vibration modes.

\begin{tabular}{|c|c|c|c|}
\hline \multirow{2}{*}{$\begin{array}{c}\text { Beam } \\
\text { Model }\end{array}$} & Classical & \multicolumn{2}{|c|}{ Improved Model } \\
\cline { 3 - 4 } & & ANSYS & COMSOL \\
\hline EBM [1] & 226.14980 & 250.3500 & 250.3487 \\
\hline RBM & 255.19065 & 279.0500 & 279.0760 \\
\hline
\end{tabular}

Where, $L$ is the beam length. The wave numbers $a_{n}^{r}$ and $b_{n}^{r}$ are calculated numerically using the expressions given in reference [2]. $Y$ and $\rho$ are respectively the effective Young's modulus and the density of the beam material. $\alpha, \beta$ and $\gamma$ are corrective factors corresponding to the beam width, the cross-section shape and the under-etching effects. They are given by the following equations:

$$
\begin{gathered}
L^{\prime}=L(1+\gamma) \\
\beta=[(I / A) /(H 2 / 12)]-1 \\
Y=E\left[1+\alpha v^{2} /\left(1-v^{2}\right)\right] .
\end{gathered}
$$

Where $v$ is the Poisson's ratio, $L^{\prime}$ is the equivalent beam length which produces the same frequencies as a microbeam with under-etching. The ratio of cross-section (with arcs of circles) to the inertia moment $(I / A)$ is calculated and it is given in reference [1].

\section{FEM Analysis and experimental validation}

The measured and extracted frequencies presented below (Tables $1 \& 2$ ) correspond to a $\mathrm{Cr}$ microbeam (size $80 \times 2 \times 0.95 \mu \mathrm{m}^{3}$ ) fabricated using the same process described in reference [3]. The microbeam having a cross-section with arcs of circles and an under-etching length measured by SEM about $3 \mu \mathrm{m}$. The results cited in reference [1] show that the extracted value of the effective Young's modulus from improved Euler-Bernoulli beam model $(\mathrm{EBM})(250.35 \mathrm{GPa})$ is very away from the value of bulk chromium (279 GPa). However, the extracted value of the effective Young's modulus from improved $\mathrm{RBM}(279.1 \mathrm{GPa})$ is very close to the measured value. These result conforms that the RBM model is more suitable to investigate dynamic behavior when $L^{2}(A / I)>10^{4}$ due to the importance of rotation effect of cross-section [2].

\section{Conclusions}

The improved RBM model is more suitable to properly investigate the dynamic behavior of thin chromium layer. The 3D FEM Analysis has been used to investigate the manufacturing process defects. The proposed corrective factors have been successfully applied and the extracted value of Young's modulus was very close to the measured values and it is about $279.1 \mathrm{GPa}$. 
FDMDII - JIP 2014

\section{References}

[1] R. Yahiaoui, A. Bosseboeuf, Therm. Mech. Simulat. Exp. Microelectron. Microsyst. EuroSimE. Proc. 5th Int. Conf. IEEE 377-384 (2004)

[2] S.M. Han, H. Benaroya, T. Wie, Eur. J. Sound. Vib. 225 (5) 935-988 (1999)

[3] R. Yahiaoui, Ph.D. thesis, University of Paris XI, Paris (2002) 